\title{
Périurbanisation et accentuation des logiques ségrégatives en Île-de-France
}

\author{
Martine Berger*
}

Alors que le constat d'une accentuation des logiques ségrégatives dans l'espace résidentiel des grandes métropoles européennes est bien établi, les pavillons périurbains ont représenté dans le dernier quart du $\mathrm{XX}^{\mathrm{e}}$ siècle un véritable modèle interclassiste auquel pratiquement tous les groupes sociaux ont accédé. En ce sens, ils ont contribué à démocratiser l'accès au confort du logement, comme l'avaient fait, en leur temps, les grands ensembles, dont la dévalorisation actuelle découle largement des processus de peuplement [Chamboredon et Lemaire, 1970]. Produit pour l'essentiel dans le cadre d'un système presque aussi industrialisé que les grands ensembles suburbains, le parc pavillonnaire périurbain risque-t-il de devenir lui aussi un des lieux phares de la question sociale ? Si on ne parle plus guère des «chalandonnettes», de nombreux travaux ont pointé les risques qui pèsent sur les ménages les plus isolés dans les espaces périurbains les moins accessibles ${ }^{1}$. Cependant l'image du périurbain pavillonnaire est plutôt celle d'un espace où dominent les classes moyennes salariées, où l'on est bien loin de l'ampleur des processus de ségrégation résidentielle et des contrastes sociaux à quelques blocs ou quelques îlots de distance qui caractérisent les tissus urbains denses du cœur des grandes métropoles.

Pourtant, la périurbanisation participe à l'exacerbation des logiques ségrégatives, pour au moins trois raisons. La constitution des quartiers pavillonnaires périurbains, surtout lorsqu'il s'agit des nouveaux villages clés en main (qui ont

* Professeur à l'université Paris-I.

1. Il y a vingt ans déjà, Odile Benoît-Guilbot [1982, 1986] décrivait les échecs de l'accession à la propriété à quelques kilomètres de Cergy, à Jaricourt (c'est-à-dire à Menucourt), ou à Saint-Cevry, près du rond-point de Gency. 
représenté près d'un tiers de la production pavillonnaire en Île-de-France depuis la fin des années 1960), relève de la volonté de constituer des communautés de pairs: c'est l'une des manifestations du refus de la mixité sociale. Les espaces périurbains sont eux-mêmes cloisonnés par des ségrégations : au départ, souvent, de microségrégations, entre opérations voisines de standing différent, entre acheteurs de modèles de différentes tailles ou construits sur des parcelles de différentes superficies. Mais de plus en plus, au fur et à mesure qu'elles se densifient, les couronnes périurbaines s'organisent selon un dispositif en auréoles et en secteurs qui prolonge les formes de division de l'espace social de l'agglomération centrale. La périurbanisation d'une partie des couches moyennes, voire modestes, a joué un rôle essentiel dans la «ghettoïsation» des grands ensembles d'habitat social et l'accélération de la «gentrification» de la capitale et de certaines communes de banlieue. Les 600000 pavillons construits en trente ans ne représentent qu'un huitième de l'offre de logements franciliens, mais leur mise sur le marché a conduit à une redistribution très ample des ménages. Les départs vers les pavillons périurbains ont créé des chaînes de vacance conduisant à la réaffectation de pans entiers du parc de logements dans l'agglomération centrale. Ils ont privé de nombreuses communes de banlieue de leurs éléments les plus jeunes, les plus actifs, les plus porteurs d'ascension sociale intergénérationnelle et contribué à la paupérisation de secteurs de l'agglomération perdant souvent la fraction la plus solvable de leurs habitants comme à la poursuite de la «gentrification» de quartiers du centre-ville ou des banlieues les mieux cotées.

\section{Périurbanisation et division sociale de l'espace métropolitain parisien}

Lorsque la périurbanisation s'amorce, à la fin des années 1960, elle conduit plutôt à une réduction des spécialisations sociales des communes rurales franciliennes, avec une diversification à la fois «passive» (effacement relatif de certaines catégories socioprofessionnelles autrefois dominantes, comme les agriculteurs ou les retraités) et «active» (diffusion dans l'ensemble de l'espace rural et périurbain de catégories en forte croissance telles que les cadres et les classes moyennes). Dans une auréole intermédiaire de communes de taille moyenne (de 500 à 2000 habitants), la première phase d'exurbanisation a accueilli un assez large éventail social de nouveaux habitants. Une densité encore faible, un éclatement fréquent en différents noyaux bâtis souvent relativement éloignés sur le territoire de la commune, rendaient la cohabitation sociale acceptable. Les communes rurales de cette deuxième couronne périurbaine n'avaient pas alors d'image de marque sociale forte, juxtaposant plutôt des microségrégations à l'intérieur du territoire communal. Par contre, le marquage social était déjà beaucoup plus net à proximité de l'agglomération parisienne. 
Progressivement, la densification de la première puis de la deuxième couronnes périurbaines, dans les années 1970 et au début des années 1980, a conduit à une spécialisation sociale croissante des périphéries. Le gradient social en fonction de la distance à Paris devient de plus en plus net au fil du temps, en même temps que l'opposition sud-ouest/nord-est se radicalise (tableau 1). Plus on s'éloigne du centre de l'agglomération, plus la part des catégories modestes augmente: contrairement au modèle classique des métropoles nord-américaines, le schéma en auréoles concentriques selon la distance à Paris renvoie tout autant, en Île-de-France, aux caractéristiques sociales de l'espace qu'à la structure démographique des ménages. Il se conjugue avec un dispositif sectoriel en quadrants qui englobe les villes petites et moyennes comme les communes rurales. À une même distance de Paris, il n'existe plus guère désormais de différence, entre espaces urbains et périurbains, en ce qui concerne la composition sociale des populations résidentes, exception faite des ménages d'employés, qui restent plus nombreux en ville, où l'offre locative est plus abondante. Dans les années 1990, tandis qu'une nouvelle ceinture ouvrière se constitue à l'est de l'Île-de-France, certains secteurs,

TABLEAU 1 - LA DISTANCE À PARIS (EN KM) DES PAVILLONS EN PROPRIÉTÉ SELON LA CATÉGORIE SOCIOPROFESSIONNELLE DE LA PERSONNE DE RÉFÉRENCE DU MÉNAGE

\begin{tabular}{|l|l|l|l|}
\hline & 1982 & 1990 & 1999 \\
\hline \multicolumn{1}{|c|}{ Ensemble } & 24,9 & 25,8 & 26,5 \\
Patrons industrie + commerce & 23,9 & 24,8 & 25,7 \\
Cadres, prof. intellectuelles supérieures & 23,5 & 24,0 & 24,2 \\
Professions intermédiaires & 25,2 & 26,6 & 27,6 \\
Contremaitres, agents de maîtrise & 26,2 & 27,6 & 28,8 \\
Employés, personnels de service & 24,8 & 26,6 & 28,0 \\
Ouvriers qualifiés & 26,3 & 28,4 & 29,5 \\
Ouvriers non qualifiés & 28,5 & 29,8 & 29,3 \\
Retraités, anciens patrons ind. et comm. & 25,4 & 26,7 & 26,3 \\
Retraités, anciens cadres & 21,7 & 22,5 & 23,6 \\
Retraités, anciennes prof. intermédiaires & 22,5 & 23,9 & 25,2 \\
Retraités, anciens employés & 24,8 & 23,8 & 24,9 \\
Retraités, anciens ouvriers & 23,8 & 25,9 & 27,0 \\
\hline
\end{tabular}

Sources : RGP 1982 et 1990 (1/4), 1999 (1/20). 
situés principalement à l'ouest, se valorisent fortement et accueillent de plus en plus de ménages de cadres. Ce marquage social induit des processus de valorisation/ dévalorisation d'autant plus durables que la part des propriétaires augmente et que la mobilité se réduit.

Cependant, après un quart de siècle de périurbanisation, l'accession à la propriété pavillonnaire s'est largement répandue dans tous les groupes sociaux. Elle concerne en moyenne en 1999 près d'un ménage francilien sur quatre $(23,4 \%)$. C'est parmi les contremaîtres et agents de maîtrise qu'elle est la plus élevée (4 ménages sur 10) - apparaissant comme la manifestation de l'ascension sociale pour une catégorie souvent d'origine ouvrière qui ne peut accéder à la propriété dans le parc collectif central -, et parmi les ouvriers non qualifiés et les employés qu'elle est la plus faible (respectivement $13 \%$ et $11 \%$ des ménages), pour des raisons qui tiennent aux revenus, aux structures des ménages (nombreux jeunes célibataires ou couples sans enfants chez les employés) voire aux projets migratoires et professionnels. Un tiers environ des retraités (sauf parmi les anciens employés) sont propriétaires et, aux âges actifs, c'est le cas d'un ménage de cadres sur quatre, d'un ménage ouvrier qualifié ou profession intermédiaire sur cinq. Les villes nouvelles, qui ont offert un foncier moins coûteux, ont largement contribué à ce processus de démocratisation de la propriété pavillonnaire : de 1975 à 1990, elles ont accueilli un cinquième de la construction de pavillons neufs en Grande Couronne.

Mais cette démocratisation a ses limites : tout le monde ne devient pas propriétaire au même endroit, les positions spatiales reflètent de plus en plus les positions sociales et les voisinages sociaux se spécialisent, comme en témoigne l'évolution des coefficients de corrélation linéaire, calculés à l'échelle de la commune, entre les groupes socioprofessionnels de propriétaires de pavillons (tableau 2). Comme on pouvait s'y attendre, ils sont plus élevés dans les tissus les plus centraux et les plus denses, traduisant la vigueur des processus d'agrégation et de ségrégation. Les oppositions cadres/ouvriers et cadres/employés se renforcent dans les espaces urbains et gagnent les communes périurbaines. Les cadres s'éloignent des professions intermédiaires, ces dernières se rapprochent des employés, voire des ouvriers (dans les espaces les plus denses), tandis qu'ouvriers et employés voisinent de plus en plus souvent. Les classes moyennes sont de plus en plus exclues des communes huppées, y compris dans le périurbain, où la mixité sociale recule fortement. 
HÉRODOTE

TABLEAU 2. - CORRÉLATIONS* ENTRE LES CATÉGORIES SOCIOPROFESSIONNELLES DES PERSONNES DE RÉFÉRENCE DES MÉNAGES PROPRIÉTAIRES DE PAVILLONS EN ÎLE-DE-FRANCE PAR ZONE ET TYPE DE COMMUNE 1975-1999

\begin{tabular}{|c|c|c|c|c|c|c|}
\hline Zone & $\begin{array}{c}\text { Cadres/ } \\
\text { prof. interm }\end{array}$ & $\begin{array}{l}\text { Cadres/ } \\
\text { ouvriers }\end{array}$ & $\begin{array}{l}\text { Cadres/ } \\
\text { employés }\end{array}$ & $\begin{array}{l}\text { Employés/ } \\
\text { ouvriers }\end{array}$ & $\begin{array}{c}\text { Employés/ } \\
\text { prof. interméd. }\end{array}$ & $\begin{array}{c}\text { Ouvriers/ } \\
\text { prof. interméd. }\end{array}$ \\
\hline $\begin{array}{l}\text { Île-de-France } \\
1975 \\
1982 \\
1990 \\
1999\end{array}$ & $\begin{array}{c}0,13 \\
\text { ns } \\
-0,11 \\
-0,27\end{array}$ & $\begin{array}{l}-0,64 \\
-0,71 \\
-0,74 \\
-0,81\end{array}$ & $\begin{array}{l}-0,17 \\
-0,29 \\
-0,40 \\
-0,55\end{array}$ & $\begin{array}{l}\mathrm{ns} \\
0,26 \\
0,49 \\
0,60\end{array}$ & $\begin{array}{l}0,22 \\
0,34 \\
0,43 \\
0,52\end{array}$ & $\begin{array}{l}\mathrm{ns} \\
0,16 \\
0,29 \\
0,36\end{array}$ \\
\hline $\begin{array}{c}\text { Petite Couronne } \\
1975 \\
1999\end{array}$ & $\begin{array}{l}-0,29 \\
-0,54\end{array}$ & $\begin{array}{l}-0,77 \\
-0,92\end{array}$ & $\begin{array}{l}-0,52 \\
-0,80\end{array}$ & $\begin{array}{l}0,45 \\
0,83\end{array}$ & $\begin{array}{l}0,38 \\
0,70\end{array}$ & $\begin{array}{l}0,34 \\
0,54\end{array}$ \\
\hline $\begin{array}{l}\text { Grande Couronne } \\
\text { pôles urbains } \\
1975 \\
1999\end{array}$ & $\begin{array}{r}0,14 \\
-0,27\end{array}$ & $\begin{array}{l}-0,72 \\
-0,81\end{array}$ & $\begin{array}{l}-0,18 \\
-0,56\end{array}$ & $\begin{array}{l}0,17 \\
0,69\end{array}$ & $\begin{array}{l}0,21 \\
0,64\end{array}$ & $\begin{array}{l}\text { ns } \\
0,47\end{array}$ \\
\hline $\begin{array}{l}\text { Grande Couronne } \\
\text { espace périurbain } \\
1975 \\
\text { et rural } 1999\end{array}$ & $\begin{array}{l}0,19 \\
\text { ns }\end{array}$ & $\begin{array}{l}-0,43 \\
-0,72\end{array}$ & $\begin{array}{c}\text { ns } \\
-0,39\end{array}$ & $\begin{array}{l}\text { ns } \\
0,32\end{array}$ & $\begin{array}{l}0,22 \\
0,22\end{array}$ & $\begin{array}{c}-0,12 \\
\mathrm{~ns}\end{array}$ \\
\hline
\end{tabular}

Sources : RGP 1975 (1/5), 1982, 1990, 1999 (1/4).

Les espaces de la propriété pavillonnaire deviennent donc de plus en plus des espaces ségrégués, ce qui était l'un des objectifs recherchés par certains pavillonnaires périurbains. Au fil du temps, dans une société où la part des ménages propriétaires de leur logement augmente, le marquage social des communes et des quartiers s'accentue: plus stables que les locataires, les propriétaires occupants visent aussi la revente ou la transmission d'un patrimoine. Filtrage social par les coûts d'accès et stratégies volontaristes de propriétaires soucieux de maintenir autant qu'ils le peuvent la valeur de leur bien contribuent à créer, dans le parc pavillonnaire nouvellement construit, des espaces socialement plus homogènes que ne l'étaient les immeubles locatifs du tissu urbain ancien. Des pratiques

\footnotetext{
* Pondérées par le nombre de ménages propriétaires de pavillons dans chaque commune.
} 
ségrégatives se développent, de fait, pour protéger l'habitabilité sociale et le «bon voisinage » jugé nécessaire à l'éducation des enfants et à l'épanouissement de la famille. La périurbanisation constitue une des formes de la mise en œuvre de ces pratiques distinctives. Le succès des lotissements clés en main n'est-il pas l'envers d'une perte de repères dans un univers professionnel de plus en plus instable dans une société postindustrielle? À défaut d'une position sociale durablement assurée dans la sphère du travail, on s'achète une «position résidentielle » qui semble d'autant mieux affirmée que les voisins sont supposés être des pairs : ils ont payé, à peu de chose près, le même prix pour accéder au même mode de vie et disposent de revenus supposés équivalents. Plus largement, à l'échelle de l'ensemble de la région et non plus seulement en Grande Couronne (où ont été réalisés les quatre cinquièmes des constructions de pavillons dans les trente dernières années), l'installation périurbaine d'une partie des ménages les plus solvables a contribué à radicaliser les contrastes sociaux dans l'espace central.

\section{La périurbanisation, un transfert aux dépens de la banlieue?}

Le phénomène s'apparente assez largement aux processus de white flight décrits dans les villes nord-américaines, à cette différence près que ce n'est pas ici le centre-ville qui perd le plus de ménages des catégories moyennes et aisées (ces départs étant d'ailleurs largement compensés à Paris par des entrées de provinciaux) mais les banlieues proches. Au cours des deux dernières décennies, les migrations résidentielles sont largement responsables de la concentration des cadres à Paris et de la conquête, par les catégories les plus aisées, de véritables bastions résidentiels dans le quadrant sud-ouest de l'Île-de-France, alors que le nord-est de la région parisienne et les secteurs industriels de la vallée de la Seine à l'amont (en direction de Corbeil), comme à l'aval (vers Mantes), ont été constamment évités par les ménages les plus riches. Le desserrement périurbain a affecté tous les groupes sociaux, mais leur déploiement vers la périphérie s'est effectué selon des destinations de plus en plus liées au niveau de leurs revenus.

Les professions intermédiaires constituent aujourd'hui le groupe social le plus régulièrement réparti dans l'espace francilien, leur proportion variant peu d'une commune à l'autre. Par contre, l'exurbanisation des ménages d'employés, souvent jeunes et en début de carrière professionnelle et familiale, est restée relativement limitée: ils continuent à se concentrer dans les communes les plus urbanisées, où l'offre locative est abondante. La périurbanisation des ouvriers, comme celle des professions intermédiaires, commence dès le début des années 1970. Alors que la banlieue rouge s'écorne et se fragmente, en particulier dans le Val-de-Marne, du fait du vieillissement et du non-renouvellement des populations ouvrières dans des communes frappées par la désindustrialisation, on observe l'émergence d'une 
véritable ceinture ouvrière aux marges de l'île-de-France, de l'Essonne au Vald'Oise. Seuls les ouvriers les plus qualifiés et les ménages comportant deux actifs peuvent espérer accéder à la propriété d'un pavillon. Fortement revalorisé, le parc rural ancien a cessé de jouer le rôle d'accueil des couches modestes qu'il assumait précédemment. Si les ouvriers représentent quatre ménages sur dix parmi les nouveaux installés entre 1982 et 1990 dans les pavillons neufs en propriété à plus de $50 \mathrm{~km}$ de Paris, quatre sur cinq sont des ouvriers qualifiés et plus des deux tiers de ces ménages sont des couples biactifs ouvriers-employées. De nombreux ménages ouvriers s'installent aussi dans le Bassin parisien proche tout en travaillant en Île-de-France.

Au total, au jeu des échanges migratoires intrarégionaux, les départements de Petite Couronne ont perdu, entre 1975 et 1999, 6500 ménages de cadres et 16700 ménages de professions intermédiaires, et, contrairement à ce que l'on observe dans Paris intra-muros, cela n'a pas été compensé par le solde migratoire avec la province. Ce n'est pas seulement du départ de ces deux catégories qu'ont souffert les banlieues, en particulier celles du quadrant nord-est. On y observe également un flux de départs des catégories ouvrières les plus solvables, celles qui peuvent accéder à la propriété pavillonnaire en Île-de-France ou dans le Bassin parisien proche. L'exemple de la Seine-Saint-Denis est particulièrement parlant: département emblématique de la concentration des populations étrangères, elle perd constamment depuis 1975 des ménages de cadres et professions intermédiaires, et gagne des ouvriers et des employés dans ses échanges avec les autres départements franciliens, déséquilibre encore accentué par le solde migratoire avec la province, l'étranger et les DOM-TOM.

Si on analyse les destinations des ménages qui ont quitté la Seine-Saint-Denis mais sont restés dans le bassin d'emploi francilien, on remarque qu'ouvriers qualifiés, employés de la fonction publique et professions intermédiaires vont s'installer dans des quartiers moins stigmatisés du Val-de-Marne ou des Hautsde-Seine, dans le pavillonnaire périurbain du Val-d'Oise et de la Seine-et-Marne, voire de l'Oise ou de l'Aisne. Ils y sont d'autant plus incités que leurs emplois se déplacent vers de nouvelles zones d'activité périphériques. Ces déplacements prennent au fil du temps une importance croissante: entre 1968 et 1975, un ménage sur cinq quittant la Seine-Saint-Denis devenait propriétaire d'un pavillon en Grande Couronne; dans les années 1980, c'est le cas d'un ménage sur quatre. Si l'on y ajoute les installations dans le Bassin parisien d'actifs conservant un emploi en Île-de-France, ce mouvement a concerné, entre 1982 et 1990, plus d'un ouvrier qualifié migrant sur trois, un contremaître sur deux, trois ménages de professions intermédiaires sur dix. De 1990 à 1999, les départs vers le parc pavillonnaire en propriété en Grande Couronne ou dans le proche Bassin parisien restent nombreux, en particulier pour les contremaîtres et agents de maîtrise, même si 
l'offre de logements neufs diminue, alors que les cadres sont de plus en plus attirés par des logements plus centraux, tout comme les employés, plus souvent célibataires, jeunes, en début de carrière professionnelle et familiale. Dans la mesure où les départs affectent davantage les catégories les plus aisées - qui sont aussi les plus mobiles -, ils ont contribué au maintien, voire au renforcement, des positions des ouvriers là où ils étaient déjà les plus nombreux. La périurbanisation des ouvriers, contremaîtres et professions intermédiaires est ainsi à l'origine de chaînes de vacance aboutissant à l'installation des familles immigrées dans le parc social délaissé par les ménages les plus solvables.

L'aire de recrutement de la main-d'œuvre de l'usine Renault de FlinsAubergenville constitue un autre exemple significatif du creusement des inégalités entre espaces périurbains et banlieues : déjà étudié par M. Guillon [1988], il est particulièrement démonstratif de la dualité croissante de la classe ouvrière francilienne. L'usine de Flins comptait encore en 1990 plus de 10000 emplois, dont trois quarts d'ouvriers. La main-d'œuvre ouvrière se composait alors pour moitié d'étrangers et de Français par acquisition; deux ouvriers sur trois étaient qualifiés, mais le niveau de qualification opposait Français et étrangers (tableaux 3a et b). De 1990 à 1999, alors que les effectifs ont diminué de plus d'un tiers, les étrangers ne représentent plus qu'un tiers de la main-d'œuvre et l'écart entre Français et étrangers demeure : $85 \%$ des ouvriers français, mais seulement la moitié des ouvriers étrangers, sont qualifiés. Les localisations résidentielles des ouvriers français et étrangers de l'usine de Flins sont radicalement différentes et leurs évolutions au cours des deux dernières décennies divergent. Plus de la moitié des français sont propriétaires d'un pavillon, très souvent hors Île-de-France : en 1999, trois ouvriers français sur dix résident hors Île-de-France et, parmi eux, huit sur dix sont propriétaires d'un pavillon. Par contre, la moitié des étrangers sont locataires dans le parc social en Grande Couronne, très peu résident hors Îlede-France, et, à l'exception des Italiens ou des Portugais, ils sont presque totalement absents de l'espace périurbain. Si un ouvrier français sur quatre est logé en HLM, il s'agit beaucoup plus souvent de cités plus petites, moins stigmatisées que ne le sont les grands ensembles de Mantes-la-Jolie ou des Mureaux.

Cette dualisation croissante caractérise l'ensemble du groupe des ouvriers franciliens. Au cours des années 1980, l'abandon du parc social et l'accession à la propriété des ménages les plus qualifiés, là où les valeurs foncières le leur permettaient, se sont accélérés. La périurbanisation des couches moyennes et modestes françaises ouvre ainsi la voie à l'entrée des familles de travailleurs immigrés dans les HLM des banlieues, au moment où elles répondent aux critères d'attribution pour ce type de logements : ancienneté suffisante de la résidence ou de l'emploi dans la commune ou dans l'entreprise, regroupement familial et transformation des structures des ménages étrangers, taux élevé de familles nombreuses. 
HÉRODOTE

TABLEAU 3A. - LES OUVRIERS FRANÇAIS ET ÉTRANGERS DE L'USINE RENAULT DE FLINS

\begin{tabular}{|l|c|c|c|c|}
\hline \multirow{2}{*}{ Nationalité } & \multicolumn{2}{c|}{ Effectif } & \multicolumn{2}{c|}{ Part relative } \\
\cline { 2 - 5 } & 1990 & 1999 & 1990 & 1999 \\
\hline Ensemble & 7736 & 4688 & & \\
Français & 4056 & 3112 & 52,1 & 66,4 \\
Dont Français de naissance & 3712 & 2804 & 47,6 & 59,8 \\
Dont Français par acquisition & 344 & 308 & 4,5 & 6,6 \\
Étrangers & 3680 & 1576 & 47,9 & 33,6 \\
\hline
\end{tabular}

Sources: RGP 1990 et $1999(1 / 4)$.

TABLEAU 3B. - LES OUVRIERS FRANÇAIS ET ÉTRANGERS DE L'USINE RENAULT DE FLINS

\begin{tabular}{|l|c|c|c|c|c|}
\hline \multicolumn{1}{|c|}{ Nationalité } & $\begin{array}{c}\text { \% ouvriers } \\
\text { qualifiés }\end{array}$ & $\begin{array}{c}\text { \% résidant hors } \\
\text { Ile-de-France }\end{array}$ & $\begin{array}{c}\text { \% résidant } \\
\text { en HLM }\end{array}$ & $\begin{array}{c}\text { \% logeant } \\
\text { en foyer }\end{array}$ & $\begin{array}{c}\text { \% propriétaires } \\
\text { de pavillons }\end{array}$ \\
\hline Ensemble 1990 & 62,1 & 16,9 & 35,7 & 6,9 & 34,1 \\
1999 & 74,6 & 22,1 & 35,1 & 3,6 & 43,2 \\
\hline Français 1990 & 77,4 & 26,8 & 23,7 & 0,5 & 57,6 \\
1999 & 83,9 & 29,8 & 27,9 & & 55,4 \\
\hline Étrangers 1990 & 41,2 & 4,9 & 49,2 & 13,8 & 9,1 \\
1999 & 55,7 & 6,8 & 49,2 & 10,4 & 19,0 \\
\hline
\end{tabular}

Sources : RGP 1990 et $1999(1 / 4)$.

La périurbanisation a donc contribué, de fait, à une concentration croissante des ménages étrangers dans le parc social des communes les plus ouvrières de banlieue : en Seine-Saint-Denis, ils constituent en $199921 \%$ des locataires HLM, au lieu de $15 \%$ en 1982.

Ceci se produit dans un contexte où, depuis trois décennies, le volume de l'offre neuve (même s'il est insuffisant eu égard aux besoins) a été largement supérieur à la croissance de la population francilienne: en vingt-cinq ans, la population a augmenté de $11 \%$, le parc de logements de $22 \%$. La relative mixité résidentielle des banlieues et des grands ensembles des années 1960 correspondait à une situation de grande pénurie de l'offre de logements, y compris pour les couches moyennes. 
Subie plutôt que choisie, elle n'a pas résisté à la détente progressive du marché immobilier: le parc social s'est trouvé progressivement écrêté par le départ de ses occupants les plus solvables vers les copropriétés ou les quartiers pavillonnaires. L'offre pavillonnaire a constitué une réponse, plébiscitée par les ménages, à leur souhait de logements plus vastes, mais aussi de différenciation sociale, de distinction [Bourdieu et al., 1990].

\section{Poursuite ou tassement de l'expansion pavillonnaire périurbaine?}

La reprise récente de la construction pavillonnaire montre que le modèle est toujours attractif pour les catégories moyennes et modestes. Il l'est aussi pour les cadres, même si ces derniers cherchent de plus en plus souvent à rester en zone dense en achetant dans l'ancien en Petite Couronne ou en Grande Couronne urbaine, ou dans la première couronne périurbaine où la mobilité est importante, les reventes nombreuses. Si la construction neuve est aujourd'hui moins diffuse que dans les années 1970, la déconcentration des emplois, qui stabilise la distance des navettes d'une partie des actifs périurbains, entretient la poursuite de l'étalement, en particulier pour les moins fortunés. Une juste mesure du desserrement résidentiel des ouvriers et employés franciliens suppose en effet qu'on prenne en compte une partie des départs vers le proche Bassin parisien, où se sont installés de nombreux actifs conservant un emploi dans la région parisienne. Or ces flux ne diminuent guère: de 1982 à 1990, ils concernaient en moyenne 5600 actifs chaque année, ils se maintiennent autour de 5300 entre 1990 et 1999. En un quart de siècle, le nombre d'actifs résidant dans le proche Bassin parisien et travaillant en Île-de-France a été plus que multiplié par trois et ils représentent désormais un dixième des actifs des huit départements bordiers. Dans une couronne de cantons jouxtant l' $̂$ le-de-France, un actif sur trois en moyenne va y travailler, et c'est le cas de quatre périurbains sur dix.

La baisse sensible de la construction pavillonnaire aux marges de l'îlede-France dans les années $1990^{2}$ ne s'accompagne pas d'un effondrement des migrations résidentielles des Franciliens. En effet, comme en Île-de-France, le desserrement périurbain ne s'y appuie pas seulement sur l'offre de logements neufs, mais aussi sur la réoccupation de l'habitat rural ancien. Dans les années 1980, quatre cinquièmes des actifs quittant l'Île-de-France pour un des départements limitrophes et continuant à travailler dans la région capitale devenaient propriétaires

2. Dans les huit départements du Bassin parisien limitrophes de l'Île-de-France, on a construit en moyenne chaque année 10000 pavillons entre 1990 et 1999, au lieu de 16600 entre 1982 et 1990, soit une baisse d'environ $40 \%$. 
d'une maison individuelle, la moitié s'installaient dans un logement neuf. Au cours de la dernière décennie, plus de deux actifs sur trois se sont dirigés vers le parc ancien ou récent et la moitié seulement ont fait l'acquisition d'un pavillon. Il s'agit pour l'essentiel de ménages modestes : employés, ouvriers et contremaîtres représentaient, en 1990, $59 \%$ des personnes de référence actives venues d'île-deFrance et y travaillant; en 1999, ils constituent encore près de la moitié (44\%) des nouveaux installés.

La concurrence pour l'accession à la propriété pavillonnaire est particulièrement vive dans les cantons qui jouxtent l'île-de-France, où plus d'un tiers $(36 \%)$ des périurbains sont de nouveaux installés venus d'Île-de-France, plus d'un sur quatre $(28,3 \%)$ est nouveau propriétaire d'un pavillon. Cette arrivée de Franciliens, souvent plus aisés que la population d'origine, pèse sur le marché foncier qu'elle tire à la hausse. Mais la concurrence est également de plus en plus forte entre les nouveaux arrivants modestes et les ménages appartenant aux professions intermédiaires, de plus en plus nombreux à quitter l'île-de-France où l'on a peu construit au cours de la dernière décennie. La déconcentration lente mais réelle des emplois du pôle parisien, le développement de nouveaux pôles à la périphérie de l'agglomération parisienne, entretiennent ce mouvement, en réduisant la distance à une gamme d'emplois de plus en plus large.

Autour de ces pôles, par exemple autour des villes nouvelles de CergyPontoise et de Saint-Quentin-en-Yvelines (qui comptent désormais chacune plus de 80000 emplois), on peut observer la constitution de ceintures périurbaines. On s'intéresse ici aux actifs qui ont déménagé de Cergy-Pontoise vers une commune rurale ou une petite ville ${ }^{3}$ au cours de la dernière période intercensitaire, les seuls pour lesquels les liens entre mobilité résidentielle et navette puissent être repérés (tableau 4). On laisse donc de côté des migrants résidentiels plus anciens, dont certains continuent à travailler en ville nouvelle, mais également ceux qui ont quitté Cergy-Pontoise et n'y travaillaient pas. La distribution sociale des actifs - et, plus encore, des ménages - reflète la décroissance des valeurs foncières avec la distance à la ville nouvelle. Les cadres choisissent de plus en plus souvent de rester près de la ville nouvelle et eux seuls ont les moyens d'assumer des coûts fonciers et immobiliers de plus en plus élevés du fait de la proximité des emplois mais aussi des commerces, des services et des équipements de la ville nouvelle. Ainsi, au fil du temps, on voit évoluer le dispositif des navettes: dans les

3. En Île-de-France, on a retenu uniquement les actifs installés dans des communes de moins de 5000 habitants en 1990, ce qui exclut par exemple Auvers et Méry-sur-Oise, ainsi que Magny-en-Vexin ou Parmain et L'Isle-Adam. Dans le Bassin parisien, trois quarts à quatre cinquièmes des actifs résident dans des communes rurales ou des unités urbaines de moins de 5000 habitants, la quasi-totalité habitant dans des villes de moins de 50000 habitants. 
années 1970 , les distances moyennes parcourues étaient plus faibles pour les catégories les moins qualifiées et les plus féminisées. Dans les années 1980, les ménages les plus aisés sont à même de s'assurer une plus grande proximité aux emplois, les plus modestes sont contraints de s'éloigner s'ils souhaitent accéder à la propriété d'un pavillon.

TABLEAU 4. - ACTIFS RÉSIDANT EN ÎLE-DE-FRANCE DANS UNE COMMUNE DE MOINS DE 5000 HAB. OU DANS LE BASSIN PARISIEN PROCHE (TOUTES COMMUNES) AYANT QUITTÉ LA VILLE NOUVELLE DE CERGY-PONTOISE ET Y TRAVAILLANT

4A. - TYPES DE MÉNAGES ET DE LOGEMENTS

\begin{tabular}{|c|c|c|c|}
\hline & 1982 & 1990 & 1999 \\
\hline $\begin{array}{l}\text { Nombre d'actifs } \\
\text { dont Île-de-France } \\
\text { dont } 8 \text { départements du Bassin parisien }\end{array}$ & $\begin{array}{l}960 \\
604 \\
356\end{array}$ & $\begin{array}{c}1564 \\
1000 \\
564\end{array}$ & $\begin{array}{rl}2 & 209 \\
1347 \\
862\end{array}$ \\
\hline $\begin{array}{l}\text { Type d'espace (délimitation 1990) (en \%) } \\
\text { pôle urbain } \\
\text { espace périurbain } \\
\text { espace multipolarisé } \\
\text { espace rural }\end{array}$ & $\begin{array}{r}13,8 \\
78,8 \\
2,9 \\
4,6\end{array}$ & $\begin{array}{r}7,9 \\
80,3 \\
2,1 \\
9,7\end{array}$ & $\begin{array}{r}8,7 \\
77,8 \\
3,2 \\
10,4\end{array}$ \\
\hline $\begin{array}{l}\text { Type de ménage } \\
\% \text { de ménages ayant des enfants } \\
\% \text { de ménages ayant } 2-3 \text { enfants } \\
\% \text { de couples biactifs }\end{array}$ & $\begin{array}{l}85,4 \\
48,0 \\
86,3\end{array}$ & $\begin{array}{l}85,9 \\
57,5 \\
79,5\end{array}$ & $\begin{array}{l}75,0 \\
52,3 \\
82,1\end{array}$ \\
\hline $\begin{array}{l}\text { Type de logement } \\
\% \text { maisons individuelles en propriété } \\
\% \text { de logements neufs }\end{array}$ & $\begin{array}{l}86,3 \\
56,7\end{array}$ & $\begin{array}{l}90,8 \\
46,3\end{array}$ & $\begin{array}{l}84,8 \\
30,3\end{array}$ \\
\hline $\begin{array}{l}\text { CSP des actifs } \\
\% \text { de cadres } \\
\% \text { de professions intermédiaires } \\
\% \text { d'employés, personnels de service } \\
\% \text { d'ouvriers }\end{array}$ & $\begin{array}{r}8,8 \\
29,6 \\
36,3 \\
21,7\end{array}$ & $\begin{array}{l}11,5 \\
27,6 \\
34,0 \\
23,0\end{array}$ & $\begin{array}{l}14,0 \\
32,1 \\
35,1 \\
16,3\end{array}$ \\
\hline $\begin{array}{l}\text { CSP des personnes de référence des ménages } \\
\% \text { de cadres } \\
\% \text { de professions intermédiaires } \\
\% \text { d'employés, personnels de service } \\
\% \text { d'ouvriers }\end{array}$ & $\begin{array}{l}13,3 \\
33,3 \\
15,0 \\
31,3\end{array}$ & $\begin{array}{l}15,6 \\
30,7 \\
13,3 \\
33,5\end{array}$ & $\begin{array}{l}21,3 \\
33,6 \\
13,9 \\
24,6\end{array}$ \\
\hline
\end{tabular}


HÉRODOTE

4B. - DiSTANCE À LA VILLE NOUVELLE SELON LA CATÉGORIE SOCIOPROFESSIONNELLE

\begin{tabular}{|l|c|c|c|c|c|c|c|c|c|}
\hline & \multicolumn{3}{|c|}{ Moyenne } & \multicolumn{3}{c|}{ Médiane } & \multicolumn{3}{c|}{$3^{\text {e }}$ quartile } \\
\cline { 2 - 11 } & 1982 & 1990 & 1999 & 1982 & 1990 & 1999 & 1982 & 1990 & 1999 \\
\hline Ensemble & 17,9 & 19,8 & 20,5 & 14,4 & 16,3 & 17,7 & 23,8 & 26,6 & 27,5 \\
\hline Selon la CSP des actifs & & & & & & & & & \\
Cadres & 19,2 & 14,5 & 17,3 & 17,0 & 11,8 & 15,3 & 30,9 & 16,6 & 25,0 \\
Professions intermédiaires & 19,2 & 16,9 & 20,5 & 13,6 & 14,0 & 17,7 & 24,0 & 25,8 & 25,3 \\
Employés, personnel de service & 16,1 & 21,0 & 18,9 & 13,2 & 17,4 & 17,3 & 23,3 & 27,6 & 26,3 \\
Ouvriers & 17,4 & 23,8 & 24,6 & 16,9 & 19,7 & 22,2 & 24,8 & 32,3 & 33,8 \\
\hline Selon la CSP des pers. de réf. ménages & & & & & & & & & \\
Cadres & 19,7 & 14,5 & 17,1 & 16,8 & 11,2 & 16,2 & 31,6 & 16,8 & 18,4 \\
Professions intermédiaires & 18,2 & 18,7 & 18,8 & 13,2 & 16,7 & 17,7 & 22,6 & 27,1 & 24,5 \\
Employés, personnel de service & 19,1 & 21,4 & 21,8 & 17,4 & 15,1 & 25,4 & 24,0 & 33,6 & 28,8 \\
Ouvriers & 16,3 & 22,7 & 21,1 & 14,0 & 18,9 & 18,1 & 23,4 & 28,2 & 28,4 \\
\hline
\end{tabular}

Sources : RGP 1975 (1/5), 1982 et 1990 (1/4), 1999 (1/4, 1/20).

La montée en puissance de pôles d'emploi périphériques fait donc rebondir la périurbanisation et contribue d'autant plus à la mise en place de nouvelles ségrégations à l'échelle microrégionale qu'il s'agit souvent des points d'entrée du réseau de transports en commun à haute fréquence, empruntés par ceux qui vont travailler à Paris ou en Petite Couronne. Si elle freine l'augmentation des trajets domicile-travail, l'évolution vers un certain degré de polycentrisme conduit donc à une accentuation des processus de division sociale de l'espace francilien. Le marché du travail francilien évolue vers une fragmentation relative des aires de recrutement, démultipliant les concurrences pour l'accès à des positions résidentielles inégalement valorisées du fait de leurs «ressources », qu'il s'agisse de leur environnement social, de la proximité des équipements et des emplois, ou de leur accessibilité.

\section{Bibliographie}

Berger M., Les Périurbains de Paris. De la ville dense à la métropole éclatée, CNRS Éditions, Paris, 2004 (+ cédérom).

BENoÎT-Guilbot O., «Identité sociale, action collective et pouvoir résidentiel à Jaricourt»,

Cahiers de l'OCS, vol. 6, Éd. du CNRS, Paris, 1982a, p. 221-326. 
-, «Système social local et mobilité résidentielle: le cas d'un village périurbain de la région parisienne », BENOîT-GUILBOT O. (dir.), Changer de région, de métier, changer de quartier, université de Paris-X, 1982b, p. 131-143.

-, «Quartiers-dortoirs ou quartiers-villages ?», L'Esprit des lieux, Localités et changement social en France, Éd. du CNRS, Paris, 1986, p. 127-156.

Bourdieu P. (dir.), "L'économie de la maison», Actes de la recherche en sciences sociales, $\mathrm{n}^{\circ} 81-82,1990$.

ChAmboredon J.-C. et Lemaire M., «Proximité spatiale et distance sociale. Les grands ensembles et leur peuplement », Revue française de sociologie, 1, 1970, p. 3-33.

GuILlON M., «Ouvriers étrangers et français des usines Renault. Pratiques de mobilisation de main-d'œuvre », Espace, populations, sociétés, 3, 1988, p. 455-466. 